\title{
1 A multi-species co-occurrence index to avoid type II errors in null model testing
}

3 Vitalis K. Lagat ${ }^{1,2^{*}}$, Guillaume Latombe ${ }^{3}$, Cang Hui ${ }^{1,4}$

$5{ }^{1}$ Centre for Invasion Biology, Department of Mathematical Sciences, Stellenbosch

6 University, Matieland 7602, South Africa

$7{ }^{2}$ DSI-NRF Centre of Excellence in Mathematical and Statistical Sciences, Wits University,

8 Johannesburg 2000, South Africa

$9{ }^{3}$ Institute of Evolutionary Biology, School of Biological Sciences, University of Edinburgh,

10 Ashworth Laboratories, Charlotte Auerbach Road, Edinburgh, EH9 3FL, United Kingdom

$11{ }^{4}$ Biodiversity Informatics Unit, African Institute for Mathematical Sciences, Cape Town

127945 , South Africa

13

14 *Email: vitaliskim@gmail.com

15 


\section{Abstract}

17 Community structure is determined by the interplay among different processes, including

18 biotic interactions, abiotic filtering and dispersal. Their effects can be detected by comparing

19 observed patterns of co-occurrence between different species (e.g. C-score and the natural

20 metric) to patterns generated by null models based on permutations of species-by-site

21 matrices under constraints on row or column sums. These comparisons enable us to detect

22 significant signals of species association or dissociation, from which the type of biotic

23 interactions between species (e.g. facilitative or antagonistic) can be inferred. Commonly

24 used patterns are based on the levels of co-occurrence between randomly paired species. The

25 level of co-occurrence for three or more species is rarely considered, ignoring the potential

26 existence of functional guilds or motifs composed of multiple species within the community.

27 Null model tests that do not consider multi-species co-occurrence could therefore generate

28 false negatives (Type II error) in detecting non-random forces at play that would only be

29 apparent for such guilds. Here, we propose a multi-species co-occurrence index (hereafter,

30 joint occupancy) that measures the number of sites jointly occupied by multiple species

31 simultaneously, of which the pairwise metric of co-occurrence is a special case. Using this

32 joint occupancy index along with standard permutation algorithms for null model testing, we

33 illustrate nine archetypes of multi-species co-occurrence and explore how frequent they are in

34 the seminal database of 289 species-by-site community matrices published by Atmar and

35 Patterson in 1995. We show that null model testing using pairwise co-occurrence metrics

36 could indeed lead to severe Type II errors in one specific archetype, accounting for $2.4 \%$ of

37 the tested community matrices.

39 Keywords: Co-occurrence; species-by-site matrix; multi-species co-occurrence index; null

40 model test; Type II error; species association. 


\section{1. Introduction}

42 A long-standing objective of ecology has been to infer the underlying community assembly

43 processes that generate patterns of species coexistence in natural communities. These

44 processes include environmental filtering, within/cross-guild biotic interactions, dispersal and

45 disturbance (Latombe et al., 2021). One of the most commonly discussed patterns is species

46 co-occurrence, which depicts the association and dissociation of species pairs in communities

47 or in multiple sites/samples of a community (Camarota et al., 2016; Cordero and Jackson,

48 2019; D’Amen et al., 2018; Kohli et al., 2018). The level of non-random co-occurrence

49 between species pairs has been interpreted as the result of two scale-dependent community

50 assembly processes. First, a full spectrum of biotic interactions, from interference to

51 facilitation, can strongly affect the level of co-occurrence at local- and mesoscales (e.g. one

52 species can locally expel others with a similar niche requirement via limiting-similarity

53 competition, or spatially synchronize its occurrence with other facilitative species; Hess et al.,

54 2020; Meszéna et al., 2006; Schamp and Jensen, 2019). Second, environmental filtering can

55 select a subset of species (typically within a functional guild) in heterogeneous environments

56 and this process is thus considered as a major driver of species co-occurrence at meso- to

57 broad-scales (Cadotte and Tucker, 2017). Two species can therefore frequently co-occur at

58 broad spatial scales due to similar habitat requirements but locally exclude each other through

59 competition. Drift and stochasticity can further interact with these scale-dependent processes

60 to generate random patterns of co-occurrence (Hou et al., 2020; Måren et al., 2018),

61 complicating our ability to discern community assembly processes and species coexistence

62 mechanisms.

63 Testing for significant levels of species co-occurrence dates back to earlier debate on the

64 role of interspecific competition in structuring ecological communities (Cordero and Jackson,

65 2019). In his analyses of the New Guinean avifauna, Diamond (1975) detected a 
66 checkerboard pattern of co-occurrence and subsequently concluded on the role of competition

67 in driving species coexistence. Connor and Simberloff $(1979,1983)$ contested this conclusion

68 by demonstrating similar co-occurrence patterns from null models with random reshuffling of

69 species. This has led to a heated debate on the role of competition in community assembly

70 and the importance of null model testing in statistical inference (especially in ecology).

71 Despite such controversies, the use of null model testing to detect significant co-occurrence

72 has become a central piece in many ecological theories and concepts (Sanderson, 2000;

73 Veech, 2014). Resource differentiation, community assembly, ecological character

74 displacement, the meta-community concept, equilibrium versus non-equilibrium organization

75 of assemblages, and the local-regional species diversity relationship, are all notable examples

76 of such theories (Davies et al., 2007; Hunt et al., 2008; Zelezniak et al., 2015).

77 Co-occurrence has traditionally been measured and tested by considering only two

78 species at a time, i.e. by assessing if the observed frequency of co-occurrence of all possible

79 species pairs is higher or lower than expected by chance (e.g., Veech, 2013, 2014). Null

80 model expectations are determined by shuffling an occupancy community matrix of species

81 presence and absence over multiple sites/samples using a number of permutation algorithms.

82 These null models therefore assume that levels of species co-occurrence only result from

83 species relative occupancy (Gotelli, 2000; Gotelli and Sounding, 2001; Lehsten and

84 Harmand, 2006). Null model tests normally rely on indices to summarize the co-occurrence

85 patterns between species pairs in the community matrix [e.g., Stone and Roberts' (1990) C-

86 score or Sanderson's (2004) natural co-occurrence metric] (Gotelli, 2000). Since a community

87 matrix typically involves more than two species, values of pairwise metrics over all pairs of

88 species are routinely averaged in the test. While these pairwise metrics have been

89 instrumental in determining the drivers of ecological community structures, they are unable 
90 to discern any interactions for more than two species simultaneously (e.g. multiple species

91 competing for the same resources or interacting with each other; Yang and Hui, 2021).

92 Null model testing using pairwise species co-occurrence metrics are constantly facing the

93 challenge of conflating multiple contending community assembly processes (Cordero and

94 Jackson, 2019). In particular, by averaging pairwise co-occurrence metrics, strong negative

95 and positive associations between different pairs of species can cancel each other out, making

96 it difficult to identify any significant non-random patterns, consequently often incurring type

97 II statistical error. For example, the test for diffuse competition requires, intrinsically, the

98 analysis of co-occurrence among multiple species and not just pairs of species within a

99 community (Veech, 2014). Averaging pairwise co-occurrence indices does not capture

100 information on the simultaneous co-occurrence.

101 Here, we present a multi-species co-occurrence index ('joint occupancy') that quantifies

102 the occupancy of two or more species simultaneously in a community. The proposed metric

103 takes the same presence-absence community matrix commonly used as inputs but captures

104 co-occurrence patterns from two to $n>2$ species (therefore generating $n-1$ values). We

105 further derive the mean and variance of the proposed joint occupancy index to facilitate

106 testing for statistical significance in observed co-occurrence patterns. Using this metric along

107 with Gotelli's (2000) recommended sim2 permutation algorithm, we present nine possible

108 archetypes of multi-species co-occurrence patterns and show how six of them can be

109 observed in a dataset of 289 published species-by-site community matrices compiled by

110 Atmar and Patterson (1995). We show that pairwise co-occurrence metrics could lead to

111 severe type II (false negative) statistical errors during the null model test for matrices

112 characterized by one of the six obtained archetypes. As such, indices of co-occurrence

113 considering multiple species simultaneously, such as our multi-species joint occupancy index,

114 should be preferred over pairwise metrics in future null model testing. 


\section{2. Materials and Methods}

\section{2.1. Null model testing}

118 Null models have been developed to provide a reference point against which alternative

119 hypotheses or inferences should be contrasted to detect non-random structures, and to infer

120 causal relationships between ecological patterns and different community assembly

121 processes. Null models have been especially instrumental in biogeography and ecology

122 (Mora et al., 2019). Despite historical controversies about their utility (D’Amen et al., 2018),

123 null models based on permutation algorithms have continued dominating the ecology

124 literature and unearthing the solutions to many ecological problems that cannot be tested by

125 conventional statistics.

126 At the mesoscale and higher, multiple candidate processes for structuring ecological

127 communities can only be inferred from observed community patterns, including co-

128 distribution patterns, whereas processes at smaller scales can rely on experiments. Null

129 models are crucial to discern non-random patterns from those random ones under certain

130 constraints so that specific processes at play can be inferred. Using null models to detect non-

131 random patterns in ecological communities requires a permutation algorithm that produces a

132 sampling distribution for a particular metric, in the absence of a mechanism of interest, so

133 that the observed patterns can be compared with those from the sampling distribution of the

134 null model. Excluding a candidate mechanism in null model testing requires imposing

135 specific constraints on the permutation algorithm, so that the 'random' patterns produced

136 from the null model can be attributed entirely to the omission of the mechanism.

137 Nine basic permutation algorithms have been proposed (Gotelli, 2000). They range from

138 no constraint at all ( $\mathrm{sim} 1)$ to those that are highly constrained on the row and column sums 
139 of the community matrix (e.g. sim9) in generating a sampling distribution. Two algorithms

140 were recommended that can arguably avoid type II errors for co-occurrence patterns: sim2

141 (which fixes the total occupancy of each species) and sim9 (which fixes both the total

142 occupancy of each species and the richness of each site). Algorithm sim2 was found to

143 produce congruent results when used with four co-occurrence indices (C-score, CHECKER,

$144 \mathrm{COMBO}$, and $V$ ratio, respectively), while sim9 worked best with C-score.

145 We corroborate previous and recent findings (Gotelli, 2000; Korňan et al., 2019; Maestre

146 et al., 2008) on the use of sim2 permutation algorithm on the premise that total number of

147 species in an ecological community (which may or may not be influenced by their biotic

148 interactions) determine the co-occurrence structure of ecological communities (Alexander et

149 al., 2018). This means that if we were to fully attribute the co-occurrence structure of an

150 ecological community to the effects of within-community biotic interactions, species must be

151 allowed to move freely among sites but the total number of sites occupied by each species

152 must be held constant during the permutation test. Rejecting the null hypothesis could signal

153 either the role of biotic interactions or the effect of propagule fluxes in and out an open

154 ecological community; consequently, a conclusion on the significant role of biotic

155 interactions could face a type I error of false positive. In most cases, however, propagule

156 fluxes serve to nullify the community structure imposed by biotic interactions, thus failing the

157 detection on the role of biotic interactions in structuralizing co-occurrence, a type II error of

158 false negative.

159 Because permutation algorithms that modify species occupancy while keep within-site

160 richness constant (e.g., sim3 and sim9) do not alter the expected number of sites occupied

161 by different species, they are not suitable for testing mechanisms of co-occurrence. In an $i$ by

$162 n$ species-by-site community matrix, the probability of finding any given number of species

163 to co-occur in a particular site remains exactly the same for such vertical permutation 
164 algorithms that retain within-site richness. In other words, site-specific richness is not a

165 reasonable constraint in null model testing for significant co-occurrence patterns. This,

166 however, does not suggest that co-occurrence patterns are independent from the variation of

167 alpha richness between sites. For instance, the level of resources, together with biotic

168 interactions, can determine the number of species that a site can hold (i.e., alpha diversity),

169 which may in turn influence species co-occurrence (see Appendix S2 for an examination of

170 the relationship between co-occurrence and richness variation).

\section{2.2. Pairwise co-occurrence metrics}

173 One of the widely used species co-occurrence metrics is the natural metric proposed by

174 Sanderson $(2000,2004)$. This metric simply counts the number of sites occupied by two

175 species simultaneously and averages over all pairs of species. The observed value of the

176 natural metric can then be compared to the sampling distribution obtained from randomizing

177 the species-by-site matrix. Typically, if the observed value lies below the null model

178 expectations (i.e., below the lower critical value of the confidence interval), the significance

179 of negative species associations (i.e., segregations or dissociations) is confirmed, which

180 normally implies the role of inter-specific competition (Cordero and Jackson, 2019) or other

181 antagonistic interactions. The significance of positive species associations (or aggregation)

182 can be inferred if the observed value lies above the null model expectations (i.e., above the

183 upper critical value of the confidence interval), which has traditionally been used to imply the

184 working of environmental filtering (Cadotte and Tucker, 2017) but can also signal facilitation

185 or mutualism.

186 Many other pairwise co-occurrence metrics have been proposed; for instance, the number

187 of unique species combinations (Pielou and Pielou, 1968), the number of species pairs 
188 forming perfect checkerboards (Diamond, 1975) and the checkerboard score (Stone and

189 Roberts, 1990). However, these pairwise metrics can only detect patterns of co-occurrence

190 between two species in community assemblages, but not patterns of three or more species

191 occurring simultaneously in the same communities. As a result, they often suffer non-

192 detection of non-stochastic processes (type II error).

194 2.3. Multi-species joint occupancy index

195 To overcome the limitations of pairwise metrics for detecting non-random higher-order co-

196 occurrence patterns in structured community assemblages, we here propose the multi-species

197 joint occupancy index. This index describes the average number of sites jointly occupied by a

198 given number of species. To illustrate the limitations of pairwise metrics, let us consider the

199 following two hypothetical cases. Three species (A, B and C) are distributed across seven

200 sites [case (a) and (b) in Figure 1], with species A occurring in 4 sites, species B in 3 sites and

201 species $C$ in 5 sites. The joint occupancies $(J)$ for all pairs of species: $J^{\{A, B\}}, J^{\{A, C\}}$ and $J^{\{B, C\}}$,

202 are 1, 3 and 2 respectively, in both cases. However, the joint occupancy of all three species is

$203 J^{\{A, B, C\}}=1$ in case (a) but 0 in case (b). This means by considering all possible orders of

204 species combinations, the multi-species joint occupancy (defined below) can discern different

205 patterns of species co-distributions, which may not be discernible by pairwise co-occurrence

206 metrics. Consequently, all pairwise metrics either do not detect change in species distribution

207 patterns [e.g. Pielou and Pielou's (1968) metric] or they only consider two species at a time

208 for its computation [for the case of Diamond's (1975), Schluter's (1984), and Stone and

209 Roberts' (1990) indices]. We therefore need to go beyond pairwise metrics to detect higher-

210 order co-occurrence patterns and associated complex interaction structures (Dieckmann et al.,

211 2000; Mayfield and Stouffer, 2017). 
212 Given a species-by-site presence-absence matrix with $m$ rows (species) and $n$ columns

213 (sites), we can count the number of sites occupied by two or more specific species

214 simultaneously, which can be easily achieved using the concept of dot product in vector

215 calculus. For instance, to compute the number of sites occupied by $i$ out of a total of $m$

216 species simultaneously, we can characterise every species (or row) by an occupancy vector,

217 e.g. species A in Figure 1(a) has $V_{1}=(1,0,1,1,0,0,1)$, and compute the dot products of all the $i$

218 vectors. Summing the elements of the resulting vector gives the joint occupancy of $i$ species.

219 Specifically, let $V_{i}=\left(v_{i, 1}, v_{i, 2}, v_{i, 3}, \ldots, v_{i, n}\right)$ be the occupancy vector of species $i$ among $n$

220 sites. For a specific set of $i$ species, denoted as $\{i\}$, we can assess whether or not this set of

221 species occurs simultaneously in a specific site $j$ by examining whether $J_{j}^{\{i\}}=\prod_{l \in\{i\}} v_{l, j}$ is

222 equal to 1 or 0 . Joint occupancy, $J^{\{i\}}$, the number of sites occupied by this set of $i$ species

223 simultaneously, can be given by

225 where $\|\cdot\|$ is the sum of all elements of the vector produced from the dot products of the $i$

226 occupancy vectors.

227 To compute the joint occupancy of unspecified sets of species in the assemblage, we

228 need to estimate the expected value of joint occupancy. Let $S_{j}$ be the number of species at site

$229 j$ and $P_{j}^{\{i\}}$ be the probability of $i$ species simultaneously occurring in site $j$; we have

$$
P_{j}^{\{i\}}=\frac{\left(\begin{array}{c}
S_{j} \\
i
\end{array}\right)}{\left(\begin{array}{c}
m \\
i
\end{array}\right)}
$$

231 where $\left(\begin{array}{c}S_{j} \\ i\end{array}\right)$ is the number of ways of selecting $i$ species out of a total of $S_{j}$ species, and $\left(\begin{array}{c}m \\ i\end{array}\right)$ is

232 the number of ways of selecting $i$ species from the entire species assemblage $(m)$. If $i=1$,

$233 P_{j}^{\{1\}}$ represents the probability of a particular species occurring in site $j$. If $i=2, P_{j}^{\{2\}}$ 
234 represents the probability of two species co-occurring in site $j$. If $i=3, P_{j}^{\{3\}}$ represents the

235 probability of 3 species simultaneously occurring in site $j$, and so forth until $i=S_{j}$, when

$236 P_{j}^{\left\{S_{j}\right\}}$ represents the probability of site $j$ harboring $S_{j}$ species. The probability $P_{j}^{\left\{i>S_{j}\right\}}$ of site $j$

237 harboring any number of species $i>S_{j}$ simultaneously is zero. Note that the probability $P_{j}^{\{i\}}$

238 of $i$ species occurring simultaneously in site $j$ is also the expected value of joint occupancy of

$239 i$ species at site $j$.

240 Using the linearity property of expectation, and assuming the sites were sampled

241 independently, we can conclude that the average number of simultaneous occurrences of $i$

242 species (or the expected value of joint occupancy) in the entire assemblage is given by

$$
J^{\{i\}}=\sum_{j=1}^{n} P_{j}^{\{i\}}
$$

244 The number of species $i$ as superscript is referred to as the order of joint occupancy. Given

245 the expected value of joint occupancy, we could calculate the variance of joint occupancy by

246 summing up the covariance of occurrences between sites:

$$
\operatorname{var}\left(J^{\{i\}}\right)=\frac{\left(\begin{array}{c}
m \\
i
\end{array}\right)}{\left(\begin{array}{c}
m \\
i
\end{array}\right)-1} \times \sum_{j=1}^{n} \sum_{k=1}^{n}\left(P_{j k}^{\{i\}}-P_{j}^{\{i\}} P_{k}^{\{i\}}\right)
$$

248 where $P_{j k}^{\{i\}}=\left(\begin{array}{c}S_{j k} \\ i\end{array}\right) /\left(\begin{array}{c}m \\ i\end{array}\right)$ is the probability of $i$ species jointly occurring in sites $j$ and $k$, and

$249 S_{j k}$ is the number of species occurring in both sites $j$ and $k$.

250 Joint occupancy is not a single measure, but a set of measures that together provide

251 insights on multi-species co-occurrence of different orders. $J^{\{1\}}$ is the average number of sites

252 occupied by a single species (i.e., average occupancy per species). $J^{\{2\}}$ is the average number

253 of sites occupied by two species simultaneously (i.e. the natural metric of co-occurrence). $J^{\{3\}}$

254 is the average number of sites occupied by three species simultaneously, and so on. By

255 considering the number of sites occupied by multiple species simultaneously, this index can 
256 assess the contribution of the spectrum from species-rich to species-poor sites, to multi-

257 species joint occupancy, and overcomes limitations of pairwise co-occurrence indices to

258 capture more precisely the non-stochastic drivers of community assembly.

\section{2.4. Nine archetypes of multi-species co-occurrence}

261 Statistical significance of pairwise co-occurrence metrics can be established by comparing its

262 observed value to the sampling distribution of null expectations from the permutation of a

263 focal species-by-site matrix. With the multi-species joint occupancy index, we can calculate

264 the observed values and sampling distributions for a range of orders. Assuming incremental

265 changes in the positions of observed joint occupancy values with respect to the sampling

266 distributions of null expectations over the full range of orders, nine archetypes of co-

267 occurrence can be defined (Figure 2). These archetypes range from those where all observed

268 joint occupancy values lie above, within or below the null expectations for all orders (i.e.,

269 above the upper critical value of the confidence interval, within the upper and lower critical

270 values, or below the lower critical value; archetypes A1, A5 and A9), to those where the

271 positions of observed joint occupancy values differ from the null expectations only for a

272 specific range of orders (Figure 2). Each archetype is representative of unique community

273 assembly processes driving species co-occurrence (see Table 1 for interpretations and

274 possible inferences for processes of all archetypes).

275 For instance, in Figure 2 A4, the observed value of multi-species joint occupancy (dark

276 line) appears inside the null distribution for the pairwise metric (i.e., when order $i=2$ ). But

277 when a higher order of species (e.g., $i=3$ ) is used, the observed value curve bends upward

278 to reach values above the null model distribution. This would indicate the presence of non-

279 random community assembly processes for groups of multiple species, that would have been 
280 unnoticed using only a pairwise metric. Similar conclusions apply to archetypes A3, A6, and

281 A7, which all reveal different processes at low and high orders of joint occupancy (Figure 2).

282 Note that in theory, the positions of observed joint occupancy values with respect to null

283 expectations could vary in more complex ways as the joint occupancy order increases (for

284 example above null expectations for $J^{\{2\}}$, within null expectations for $J^{\{3\}}$, and above null

285 expectations again for $\left.J^{\{4\}}\right)$. Nonetheless, we expect this kind of complex configurations to be 286 rare.

\section{2.5. Community matrix and testing}

289 With the proposed joint occupancy index and sim2 permutation algorithm, we performed

290 null model testing of multi-species co-occurrence patterns for 289 species-by-site presence-

291 absence matrices compiled by Atmar and Patterson (1995). The numbers of species in these

292 communities range from 3 to 309 and the numbers of sites from 3 to 202, representing about

29340 taxonomic groups. Note, it is obvious that the number of sites harboring multiple species

294 simultaneously (thus joint occupancy, $J^{\{i\}}$ ) decreases with its order (i.e. the number of

295 species, $i$ ). To understand the forms of this decline, we fitted exponential, power law and

296 exponential-power law parametric forms to order-dependent joint occupancy. Fitting these

297 parametric models to how joint occupancy declines with orders offer additional insights to

298 forces behind species co-occurrence patterns and the role of biotic interactions. Similar to the

299 fact that the parametric form of zeta diversity decline of multisite compositional similarity

300 can unveil stochastic versus deterministic assembly processes at work in ecological

301 communities (Hui and McGeoch, 2014; McGeoch et al., 2019), we could anticipate different

302 orders of biotic interactions, other than just pairwise interactions, that are responsible to co-

303 occurrence at different orders (e.g. neutral force of random encountering versus niche-based 
304 force of functional trait matching/complementarity). As higher order joint occupancy can

305 only occur in species rich sites, we further anticipate the entangled concepts of species

306 turnover and co-occurrence.

307 Specifically, using the joint occupancy index, we calculated $J^{\{i\}}$ for an observed matrix.

308 Using sim2 permutation algorithm (which reshuffles the observed matrix while keeping

309 species occupancy constant), we calculated $J^{\{i\}}$ for 999 randomized matrices. We used these

3101000 values of $J^{\{i\}}$ as its sampling distribution, from which the $95 \%$ [closed] confidence

311 interval were used for hypothesis testing. All the results presented in this paper were

312 computed using msco R Package in R version 4.1.1 (see Appendix S1).

\section{3. Results}

315 Joint occupancy, $J^{\{i\}}$, declines monotonically with the number of species $i$. When two

316 parametric forms [exponential: $J^{\{i\}}=a \cdot \exp (b \cdot i)$; and power law: $J^{\{i\}}=a \cdot i^{b}$ ] were fitted

317 to how joint occupancy declines with increasing number of species (hereafter, "joint

318 occupancy decline'), exponential form fitted with $R^{2}>0.95$ in 257 (88.9\%) of the 289

319 community matrices and power law with the same level of the goodness-of-fit in $243(84.1 \%)$

320 cases. However, power law had lower AIC values than exponential in 55 (19\%) communities,

321 while exponential had lower AIC values than power law in $234(81 \%)$ communities. When

322 the two models were combined, the resulting model [exponential-power law: $J^{\{i\}}=a$.

$\left.323 \exp (b \cdot i) \cdot i^{c}\right]$ provided the best fit $\left(R^{2}>0.95\right.$ in 285 communities $)$. Among the three

324 models, exponential-power law provided the lowest AIC values in 273 communities, while

325 exponential and power law had the lowest AIC values in 11 and 5 communities, respectively

326 (see summary in Table 2 and full results in Appendix S1). This means the exponential-power 
327 law was the best model (among the three) in fitting joint occupancy decline in $94 \%$ of

328 communities.

329 The null model tests identified six archetypes in the 289 communities (A1, A2, A4, A5,

330 A8 and A9; see Figure 3 and Table 1 for interpretations of archetypes), ranging from those

331 that indicate positive species associations (A1) to those of negative species association (A9)

332 among species residing respective communities. Archetype A1, under which species co-

333 occurring more than expected by chance over all orders, dominated $63.7 \%$ of these

334 communities. Of these A1 communities, the exponential-power law parametric model had the

335 lowest AIC values in 98\% (180) of cases among the three parametric models. When only

336 power law and exponential forms were compared, power law dominated $27 \%$ while

337 exponential $73 \%$ of A1 cases.

338 A5 was the second most common archetype, characterizing 82 communities (28\%).

339 Under this archetype, observed co-occurrence does not differ significantly from null model

340 expectations across all orders. Exponential form fitted better than power law in $95 \%$ of A5

341 communities (Table 2). There were only 22 communities following other four archetypes

$342(\mathrm{~A} 2=11, \mathrm{~A} 4=7, \mathrm{~A} 8=1$, and A9=3; see Table 2). In particular, pairwise metrics (order 2) failed

343 to detect non-stochastic forces at work for higher-order co-occurrence in the 7 A4

344 communities (i.e. type II error of false negative; Figure 3). There was one community that did

345 not follow any of the nine archetypes ('NA' in Table 2), representing the combinations of

346 several proposed archetypes and highlighting the possibility of rare but highly complex co-

347 occurrence patterns in real communities (see community 80 in Appendix S3). 
350 Inferring underlying processes from patterns requires to either examine multiple patterns

351 capturing different pieces of information about the community structure (Grimm and

352 Railsback, 2012; Latombe et al., 2011) or an informative pattern that can reflect and bridge

353 many facets of biodiversity structure (Hui and McGeoch, 2014). The proposed multi-species

354 joint occupancy index $\left(J^{\{i\}}\right)$ is such an informative and complete set of indices for describing

355 species co-occurrences. Instead of only calculating the average over a set of pairwise (or any

356 order $i \leq m$ ) co-occurrences, we can compute the average over all sets of simultaneous

357 species occurrences, and compare the joint occupancy decline to its null form, therefore

358 capturing information on the identity of sites occupied by multiple species simultaneously.

359 Since it reckons with all possible species simultaneous occurrences, this index can be used in

360 testing for significant co-occurrence patterns among multiple species (e.g., diffuse

361 competition) within a community, contrary to pairwise co-occurrence metrics.

362 The gain of information captured by our new joint occupancy metric is apparent in the

363 nine basic archetypes of species co-occurrence we could identified, instead of three for

364 pairwise metrics. Of the six archetypes detected in the 289 matrices, archetype A1 and A5

365 were the most common ones. Archetype A5 indicates that species co-occur as expected by

366 chance when species occupancy is fixed, across all orders of joint occupancy. As archetype

367 A5 depicts species co-occurrence patterns conforming to null model expectations across the

368 full spectrum of orders, it represents the dominance of stochastic/neutral ecological processes

369 in a community. Because the exponential form of joint occupancy decline fitted better than

370 power law in $95 \%$ of A5 communities (Table 2), this particular form could signal stochastic

371 co-occurrence among species. Hui and McGeoch (2014) found that stochastic community

372 assembly can give rise to an exponential decline of compositional similarity, our results here

373 point out to the possible connection between species turnover and co-occurrence in a 
374 community: an exponential decline of compositional similarity with zeta diversity order

375 could correspond to an exponential decline of joint occupancy.

376 Archetype A1 indicates stronger species association than expected by chance across all

377 orders of joint occupancy. This suggests that habitat filtering is an important driver of

378 community assembly (Cordero and Jackson, 2019). A1 communities tend to have the largest

379 variation in their within-site richness (Figure S1), whereas A5 communities, characterized by

380 stochastic processes have some of the lowest variations in within-site richness. These results

381 corroborate previous findings that environmental filtering can enhance habitat heterogeneity

382 and consequently the gradient of species richness (Schuler et al., 2017). In contrast, archetype

383 A9 indicates stronger species segregation than expected by chance across all orders of joint

384 occupancy, signaling the work from intense inter-specific competition or other antagonistic

385 interactions. Because A1 communities are much more common than A9 communities,

386 environmental filters could be, in general, a more dominant driver of species co-occurrence

387 patterns than competition, at least at the spatial scales represented in the dataset.

388 The fact that the vast majority of datasets belongs to categories A1, A5 or A9 indicates

389 that the processes structuring a community tend to apply to all groups of species (e.g.

390 functional guilds) composing the community, regardless of their size. The other archetypes

391 observed in the dataset (A2, A7 and A8) are characteristic of communities with rarer

392 assembly processes that change for different groups of species within the communities.

393 Although they are quite rare, they represent special cases that can enable us to better

394 understand when and why community assembly processes diverge from expectations, and

395 may represent unique types of ecosystems. Archetypes A2 and A8 represent communities for

396 which non-random co-occurrence of species is only observed for low orders of joint

397 occupancy, and characterized 11 and one community, respectively. These archetypes indicate 
398 communities for which niche processes are at play, but only for part of the community,

399 between a small number of species.

400 Two of the nine archetypes (A4 and A6) depict patterns of co-occurrence different from

401 null expectations at only high orders, i.e. a type II error. Only one of these archetypes (A4)

402 was observed in the dataset, characterizing 7 of the 289 matrices. It indicates the presence of

403 complex interactions between large groups of species that are absent for small groups, an

404 aspect that is not commonly explored in community ecology. Importantly, despite being

405 relatively rare, the theoretical and empirical possibilities of encountering these archetypes

406 illustrates the importance of using an appropriate metric for null model testing of species co-

407 occurrence patterns to obtain a comprehensive perspective on community assembly enabling

408 us to identify unique ecosystems.

409 The nine archetypes are certainly not exhaustive, and more complex patterns can exist

410 (e.g. see community 80 in Appendix S3). Although there are still notable limitations to how

411 different archetypes can be interpreted (even at orders $>2$, we can still face some of the same

412 issues as pairwise indices, such as the fact that different groups of species may be driven by

413 opposing positive and negative associations that can cancel each other out, making it difficult

414 to identify any significant non-random patterns), multi-species joint occupancy nonetheless

415 provides a comprehensive description of co-occurrence patterns and represents a firm step

416 forward to better deciphering ecological processes at play in communities.

\section{Acknowledgements}

419 VKL is supported by the DSI-NRF Centre of Excellence in Mathematical and Statistical

420 Sciences (CoE-MaSS) at Wits University and the Science Faculty Scholarship at

421 Stellenbosch University. $\mathrm{CH}$ is supported by the National Research Foundation of South 
422

423

424

\section{Literature cited}

426

427

428

429

430

431

432 Cadotte, M.W., Tucker, C.M., 2017. Should environmental filtering be abandoned? Trends

434 Camarota, F., Powell, S., S. Melo, A., Priest, G., J. Marquis, R., L. Vasconcelos, H., 2016.

455 Gotelli, N.J., 2000. Null model analysis of species co-occurrence patterns. Ecology 81(9), 456 Co-occurrence patterns in a diverse arboreal ant community are explained more by competition than habitat requirements. Ecol. Evol. 6(24), 8907-8918. https://doi.org/10.1002/ece3.2606

Connor, E.F., Simberloff, D., 1983. Interspecific competition and species co-occurrence patterns on islands: Null models and the evaluation of evidence. Oikos 41(3), 455-465. https://doi.org/10.2307/3544105

Connor, E.F., Simberloff, D., 1979. The Assembly of species communities: chance or competition? Ecology 60(6), 1132-1140. https://doi.org/10.2307/1936961

Cordero, R.D., Jackson, D.A., 2019. Species $\square$ pair associations, null models, and tests of mechanisms structuring ecological communities. Ecosphere 10(7), e02797. https://doi.org/10.1002/ecs2.2797

D’Amen, M., Mod, H.K., Gotelli, N.J., Guisan, A., 2018. Disentangling biotic interactions, environmental filters, and dispersal limitation as drivers of species co-occurrence. Ecography (Cop.). 41(8), 1233-1244. https://doi.org/10.1111/ecog.03148

Diamond, J.M., 1975. Assembly of species communities. In: Diamond, J.M. and Cody, M.L., Eds., Ecology and Evolution of Communities, Harvard University Press, Boston, 342344.

Dieckmann, U. , Law, R., \& Metz, J.A.J. (2000). The Geometry of ecological interactions: simplifying spatial complexity. Cambridge: Cambridge University Press. ISBN 0521642949 
Gotelli, N.J., Sounding, E., 2001. Research frontiers in null model analysis. Glob. Ecol. Biogeogr. 10(4), 337-343. https://doi.org/10.1046/j.1466-822X.2001.00249.x

Grimm, V., Railsback, S.F., 2012. Pattern-oriented modelling: a "multi-scope" for predictive systems ecology. Philos. Trans. R. Soc. B Biol. Sci. 367(1586), 298-310. https://doi.org/10.1098/rstb.2011.0180

Hess, M.C.M., Buisson, E., Jaunatre, R., Mesléard, F., 2020. Using limiting similarity to enhance invasion resistance: Theoretical and practical concerns. J. Appl. Ecol. 57(3), 559-565. https://doi.org/10.1111/1365-2664.13552

Hou, F., Zhang, H., Xie, W., Zhou, X., Zhu, X., Zhang, D., 2020. Co-occurrence patterns and assembly processes of microeukaryotic communities in an early-spring diatom bloom. Sci. Total Environ. 711, 134624. https://doi.org/10.1016/j.scitotenv.2019.134624

Hui, C., McGeoch, M.A., 2014. Zeta diversity as a concept and metric that unifies incidencebased biodiversity patterns. Am. Nat. 184(5), 684-694. https://doi.org/10.1086/678125

Hunt, D.E., David, L.A., Gevers, D., Preheim, S.P., Alm, E.J., Polz, M.F., 2008. Resource partitioning and sympatric differentiation among closely related bacterioplankton. Science, 320(5879), 1081-1085. https://doi.org/10.1126/science.1157890

Jonathan Davies, T., Meiri, S., Barraclough, T.G., Gittleman, J.L., 2007. Species co-existence and character divergence across carnivores. Ecol. Lett. 10(2), 146-152. https://doi.org/10.1111/j.1461-0248.2006.01005.x

Kohli, B.A., Terry, R.C., Rowe, R.J., 2018. A trait-based framework for discerning drivers of species co-occurrence across heterogeneous landscapes. Ecography (Cop.). 41(12), 1921-1933. https://doi.org/10.1111/ecog.03747

Korňan, M., Svitok, M., Krištín, A., 2019. Null model analyses of temporal patterns of bird assemblages and their foraging guilds revealed the predominance of positive and random associations. Ecol. Evol. 9(15), 8541-8554. https://doi.org/10.1002/ece3.5372

Latombe, G., Parrott, L., Fortin, D., 2011. Levels of emergence in individual based models: Coping with scarcity of data and pattern redundancy. Ecol. Modell. 222(9), 1557-1568. https://doi.org/10.1016/j.ecolmodel.2011.02.020

Latombe, G., Richardson, D.M., McGeoch, M.A., Altwegg, R., Catford, J.A., Chase, J.M., Courchamp, F., Esler, K.J., Jeschke, J.M., Landi, P., Measey, J., Midgley, G.F., Minoarivelo, H.O., Rodger, J.G., Hui, C., 2021. Mechanistic reconciliation of community and invasion ecology. Ecosphere 12(2), e03359. https://doi.org/10.1002/ecs2.3359

Lehsten, V., Harmand, P., 2006. Null models for species co-occurrence patterns: Assessing bias and minimum iteration number for the sequential swap. Ecography (Cop.). 29(5), 786-792. https://doi.org/10.1111/j.0906-7590.2006.04626.x

Maestre, F.T., Escolar, C., Martínez, I., Escudero, A., 2008. Are soil lichen communities structured by biotic interactions? A null model analysis. J. Veg. Sci. 19(2), 261-266. 
https://doi.org/10.3170/2007-8-18366

496 Måren, I.E., Kapfer, J., Aarrestad, P.A., Grytnes, J.A., Vandvik, V., 2018. Changing

497 contributions of stochastic and deterministic processes in community assembly over a

498 successional gradient. Ecology 99(1), 148-157. https://doi.org/10.1002/ecy.2052

499 Mayfield, M.M., Stouffer, D.B., 2017. Higher-order interactions capture unexplained

$500 \quad$ complexity in diverse communities. Nat. Ecol. Evol. 1(3), 1-7.

501 https://doi.org/10.1038/s41559-016-0062

502 McGeoch, M.A., Latombe, G., Andrew, N.R., Nakagawa, S., Nipperess, D.A., Roigé, M.,

503 Marzinelli, E.M., Campbell, A.H., Vergés, A., Thomas, T., Steinberg, P.D., Selwood,

504 K.E., Henriksen, M. V., Hui, C., 2019. Measuring continuous compositional change

505 using decline and decay in zeta diversity. Ecology 100(11), e02832.

506 https://doi.org/10.1002/ecy.2832

507 Meszéna, G., Gyllenberg, M., Pásztor, L., Metz, J.A.J., 2006. Competitive exclusion and

508 limiting similarity: a unified theory. Theor. Popul. Biol. 69(1), 68-87.

509 https://doi.org/10.1016/j.tpb.2005.07.001

510 Mora, B.B., Dalla Riva, G. V., Stouffer, D.B., 2019. Unmasking structural patterns in

511 incidence matrices: an application to ecological data. J. R. Soc. Interface 16(151),

512 20180747. https://doi.org/10.1098/rsif.2018.0747

513 Pielou, D.P., Pielou, E.C., 1968. Association among species of infrequent occurrence: the

514 insect and spider fauna of Polyporus betulinus (Bulliard) Fries. J. Theor. Biol. 21(2),

515 202-216. https://doi.org/10.1016/0022-5193(68)90070-2

516 Sanderson, J.G., 2000. Testing ecological patterns. Am. Sci. 88(4), 332-339.

517 https://doi.org/10.1511/2000.4.332

518 Sanderson, J.G., 2004. Null model analysis of communities on gradients. J. Biogeogr. 31(6),

519 879-883. https://doi.org/10.1111/j.1365-2699.2004.01054.x

520 Schamp, B.S., Jensen, A.M., 2019. Evidence of limiting similarity revealed using a

521 conservative assessment of coexistence. Ecosphere 10(8), e02840.

522 https://doi.org/10.1002/ecs2.2840

523 Schluter, D., 1984. A variance test for detecting species associations, with some example

524 applications. Ecology 65(3), 998-1005. https://doi.org/10.2307/1938071

525 Schuler, M.S., Chase, J.M., Knight, T.M., 2017. Habitat size modulates the influence of

526 heterogeneity on species richness patterns in a model zooplankton community. Ecology

527 98(6), 1651-1659. https://doi.org/10.1002/ECY.1833

528 Stone, L., Roberts, A., 1990. The checkerboard score and species distributions. Oecologia

529 85(1), 74-79. https://doi.org/10.1007/BF00317345

530 Veech, J.A., 2013. A probabilistic model for analysing species co-occurrence. Glob. Ecol.

531 Biogeogr. 22(2), 252-260. https://doi.org/10.1111/j.1466-8238.2012.00789.x 
532 Veech, J.A., 2014. The pairwise approach to analysing species co-occurrence. J. Biogeogr.

533 41, 1029-1035. https://doi.org/10.1111/jbi.12318

534 Yang, Y., Hui, C., 2021. How competitive intransitivity and niche overlap affect spatial

535 coexistence. Oikos 130(2), 260-273. https://doi.org/10.1111/oik.07735

536 Zelezniak, A., Andrejev, S., Ponomarova, O., Mende, D.R., Bork, P., Patil, K.R., 2015.

537 Metabolic dependencies drive species co-occurrence in diverse microbial communities.

$538 \quad$ Proc. Natl. Acad. Sci. U.S.A. 112(20), 6449-6454.

539 https://doi.org/10.1073/pnas.1421834112

540 
Table 1: Archetypes of species co-occurrence patterns in ecological communities as brought out by a null model test using joint occupancy index and various permutation algorithms.

\begin{tabular}{|c|c|c|}
\hline Archetype & Description & Interpretation \\
\hline A1 & $\begin{array}{l}\text { The joint occupancy }\left(J^{\{i\}}\right) \\
\text { decreases monotonically with } \\
i \text {. Its values for the observed } \\
\text { data is above the upper critical } \\
\text { values of the } 95 \% \text { closed } \\
\text { confidence interval of the joint } \\
\text { occupancy values for the } \\
\text { simulated data (null model). }\end{array}$ & $\begin{array}{l}\text { The null hypothesis is false across all orders, with species co- } \\
\text { occurring more than by chance. This suggests the presence of } \\
\text { a number of ecological mechanisms (e.g. environmental } \\
\text { filtering or shared habitat requirements) driving community } \\
\text { structure at the spatial scale considered (Cordero and Jackson, } \\
\text { 2019). }\end{array}$ \\
\hline A2 & $\begin{array}{l}\text { The observed joint occupancy } \\
\text { values for lower and higher } \\
\text { orders }(i) \text { of species are above } \\
\text { and inside the null model, } \\
\text { respectively. }\end{array}$ & $\begin{array}{l}\text { The null hypothesis is false for lower orders, with species co- } \\
\text { occurring more than by chance, and true for higher order of } \\
\text { joint occupancy. This archetype suggests that functional guilds } \\
\text { are composed of few species co-occurring simultaneously, for } \\
\text { example due to habitat filtering, or the presence of facilitative } \\
\text { interactions between few species. }\end{array}$ \\
\hline A3 & $\begin{array}{l}\text { The observed joint occupancy } \\
\text { values for lower and higher } \\
\text { orders }(i) \text { of species are above } \\
\text { and below the null model, } \\
\text { respectively. }\end{array}$ & $\begin{array}{l}\text { Species co-occur more often than by chance in sites with few } \\
\text { species, but co-occur less often than by chance in sites with } \\
\text { many species. This implies that at least two different } \\
\text { community assembly processes govern community structure. }\end{array}$ \\
\hline A4 & $\begin{array}{l}\text { The observed joint occupancy } \\
\text { values for lower and higher } \\
\text { orders }(i) \text { of species are inside } \\
\text { and above the null model, }\end{array}$ & $\begin{array}{l}\text { The null hypothesis is true at low orders, but false at high } \\
\text { orders, with species co-occurring more often than expected by } \\
\text { chance. Pairwise co-occurrence only fails at detecting species } \\
\text { co-occurrence patterns of aggregation for sites with many } \\
\text { species, i.e. a type II error. This suggests the existence of }\end{array}$ \\
\hline
\end{tabular}




\begin{tabular}{|c|c|c|}
\hline & respectively. & complex facilitative interactions between multiple species. \\
\hline A5 & $\begin{array}{l}\text { The values of joint occupancy } \\
\text { for the observed data are } \\
\text { inside the null model, } \\
\text { implying the test is } \\
\text { statistically insignificant. }\end{array}$ & $\begin{array}{l}\text { The null hypothesis is true across all orders. This indicates } \\
\text { that the structure of ecological communities is essentially } \\
\text { driven by stochastic processes (Cordero and Jackson, 2019; } \\
\text { Gotelli, 2000; Gotelli and Sounding, 2001). }\end{array}$ \\
\hline A6 & $\begin{array}{l}\text { The observed joint occupancy } \\
\text { values for lower and higher } \\
\text { orders }(i) \text { of species are inside } \\
\text { and below the null model, } \\
\text { respectively. }\end{array}$ & $\begin{array}{l}\text { The null hypothesis is true at low orders, but false at high } \\
\text { orders, with species co-occurring less often than expected by } \\
\text { chance. As for A4, pairwise co-occurrence only fails at } \\
\text { detecting species co-occurrence patterns of aggregation for } \\
\text { sites with many species, i.e. a type II error. This suggests the } \\
\text { existence of complex competitive interactions between } \\
\text { multiple species. }\end{array}$ \\
\hline A7 & $\begin{array}{l}\text { The observed joint occupancy } \\
\text { values for lower and higher } \\
\text { orders }(i) \text { of species are below } \\
\text { and above the null model, } \\
\text { respectively. }\end{array}$ & $\begin{array}{l}\text { Species co-occur less often than by chance in sites with few } \\
\text { species, but co-occur more often than by chance in sites with } \\
\text { many species. This implies that at least two different } \\
\text { community assembly processes govern community structure. }\end{array}$ \\
\hline A8 & $\begin{array}{l}\text { The observed joint occupancy } \\
\text { values for lower and higher } \\
\text { orders }(i) \text { of species are below } \\
\text { and inside the null model, } \\
\text { respectively. }\end{array}$ & $\begin{array}{l}\text { The null hypothesis is false for lower orders, with species co- } \\
\text { occurring less than by chance, and true for higher order of } \\
\text { joint occupancy. This archetype suggests the presence of } \\
\text { strong competitive interactions between species within small } \\
\text { groups. }\end{array}$ \\
\hline A9 & $\begin{array}{l}\text { The values of joint occupancy } \\
\text { for the observed data are } \\
\text { below the null model, for all } \\
\text { orders of species. }\end{array}$ & $\begin{array}{l}\text { The null hypothesis is false across all orders, with species co- } \\
\text { occurring less than by chance. Processes such as inter-specific } \\
\text { competition or limiting similarity could be the forces behind } \\
\text { such community structure (Cordero and Jackson, 2019), albeit } \\
\text { predation may also generate similar patterns. }\end{array}$ \\
\hline
\end{tabular}


bioRxiv preprint doi: https://doi.org/10.1101/2021.11.03.467033; this version posted November 4, 2021. The copyright holder for this preprint (which was not certified by peer review) is the author/funder, who has granted bioRxiv a license to display the preprint in perpetuity. It is made available under aCC-BY-NC-ND 4.0 International license.

\section{2}


Table 2. The frequency of the six realized archetypes of species co-occurrence patterns and the parametric forms of joint occupancy decline for all communities under each archetype. The three parametric forms fit extremely well (most $R^{2}>0.95$ ) to joint occupancy decline. However, to further discern the best form, we based on AIC to select only the best form for each community. Exponential-power law provided the best fit in $94 \%$ of all communities, then exponential (4\%) and power law (2\%) respectively. When exponential and power law forms were compared exclusively, exponential was better than power law in $81 \%$ of communities, with $19 \%$ communities fitting better in power law than exponential [see Jo.res function in ms co R Package]. The "abc" values in square brackets constitute the 95\% closed confidence interval of the parameter estimates (of these regression models) for all communities under each archetype. Archetypes A3, A6, and A7 were not observed in our data.

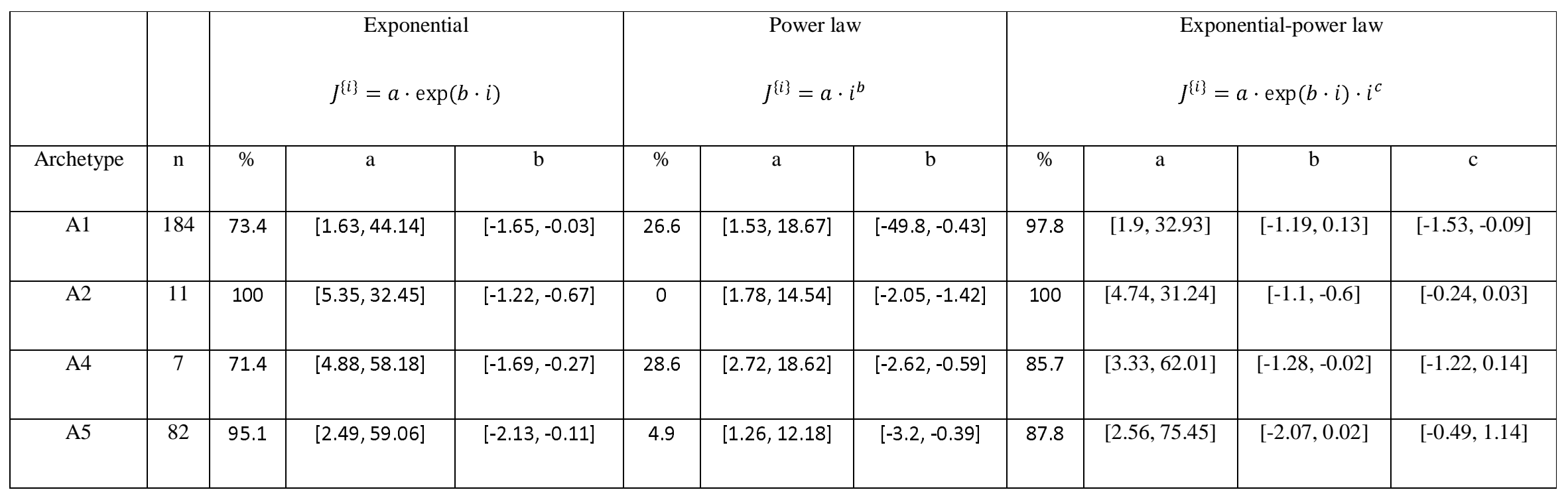




\begin{tabular}{|c|c|c|c|c|c|c|c|c|c|c|c|}
\hline A8 & 1 & 100 & {$[24.17,24.17]$} & {$[-2.29,-2.29]$} & 0 & {$[2.45,2.45]$} & {$[-3.43,-3.43]$} & 100 & {$[30.6,30.6]$} & {$[-2.53,-2.53]$} & {$[0.35,0.35]$} \\
\hline A9 & 3 & 100 & {$[8.46,81.73]$} & {$[-2.57,-0.98]$} & 0 & {$[2.35,5.96]$} & {$[-3.81,-1.79]$} & 66.7 & {$[13.72,44.01]$} & {$[-2,-1.47]$} & {$[-0.83,0.83]$} \\
\hline NA & 1 & 100 & {$[43.54,43.54]$} & {$[-1.63,-1.63]$} & 0 & {$[8.55,8.55]$} & {$[-2.56,-2.56]$} & 100 & {$[37.19,37.19]$} & {$[-1.47,-1.47]$} & {$[-0.24,-0.24]$} \\
\hline
\end{tabular}


544 Figure 1. For the two communities (a) and (b), the values of the pairwise index $(i=2)$ :

$545 J^{\{A, B\}}, J^{\{A, C\}}$ and $J^{\{B, C\}}$ are 1,3 and 2, respectively. However, when the higher order of joint

546 occupancy $\left(J^{\{A, B, C\}} ; i=3\right)$ is used, we get one for community (a) and zero for community

547 (b). This means irrespective of the two different patterns in the two communities, the pairwise

548 metric does not draw a distinction between them, unlike the higher order metric. It suffices

549 then to say multi-species joint occupancy detects change in species distribution patterns

550 across different sites while pairwise co-occurrence does not.

552 Figure 2. Nine possible archetypes of species co-occurrence patterns from the null model test 553 of the same patterns. The number of communities under each archetype are given in brackets.

554 The dark-gray ribbons represent the null model with its upper and lower boundaries being the 555 critical values of the $95 \%$ closed confidence interval of the joint occupancy values (computed 556 using eqn 3) of simulated data. Depending on the position of the black curves and lines

557 (representing the joint occupancy values [from eqn 3] of observed data) relative to the null

558 model, the test can be deemed statistically significant. As the number of species increase, the

559 results and consequently, the inferences from the null model test, change in some archetypes.

560 This is illustrated, for instance, by the gray dotted lines where two species considered

561 simultaneously lead to different ecological interpretations as to what structures ecological

562 communities compared to when three or more species are considered simultaneously.

564 Figure 3. Archetypes of species co-occurrence patterns from the 289 empirical data matrices 565 analyzed. The number of sites occupied by multiple species simultaneously (joint occupancy, $566 J^{\{i\}}$; obtained from eqn 3$)$ declines monotonically as the number of species $(i)$ increase in all 567 the archetypes. They range from communities whose species co-occurrences are positively 
568 associated more often than would be expected by chance (aggregation), to those that are

569 negatively associated more often than by chance if species colonized sites randomly

570 (segregation). A4 is typical of a community where a pairwise metric fails at detecting

571 deterministic species co-occurrence patterns leading to type II statistical error. By using a

572 multi-species joint occupancy index, the contributions made by the associations between

573 multiple species towards the structure of ecological communities can be reckoned with. 
Sites

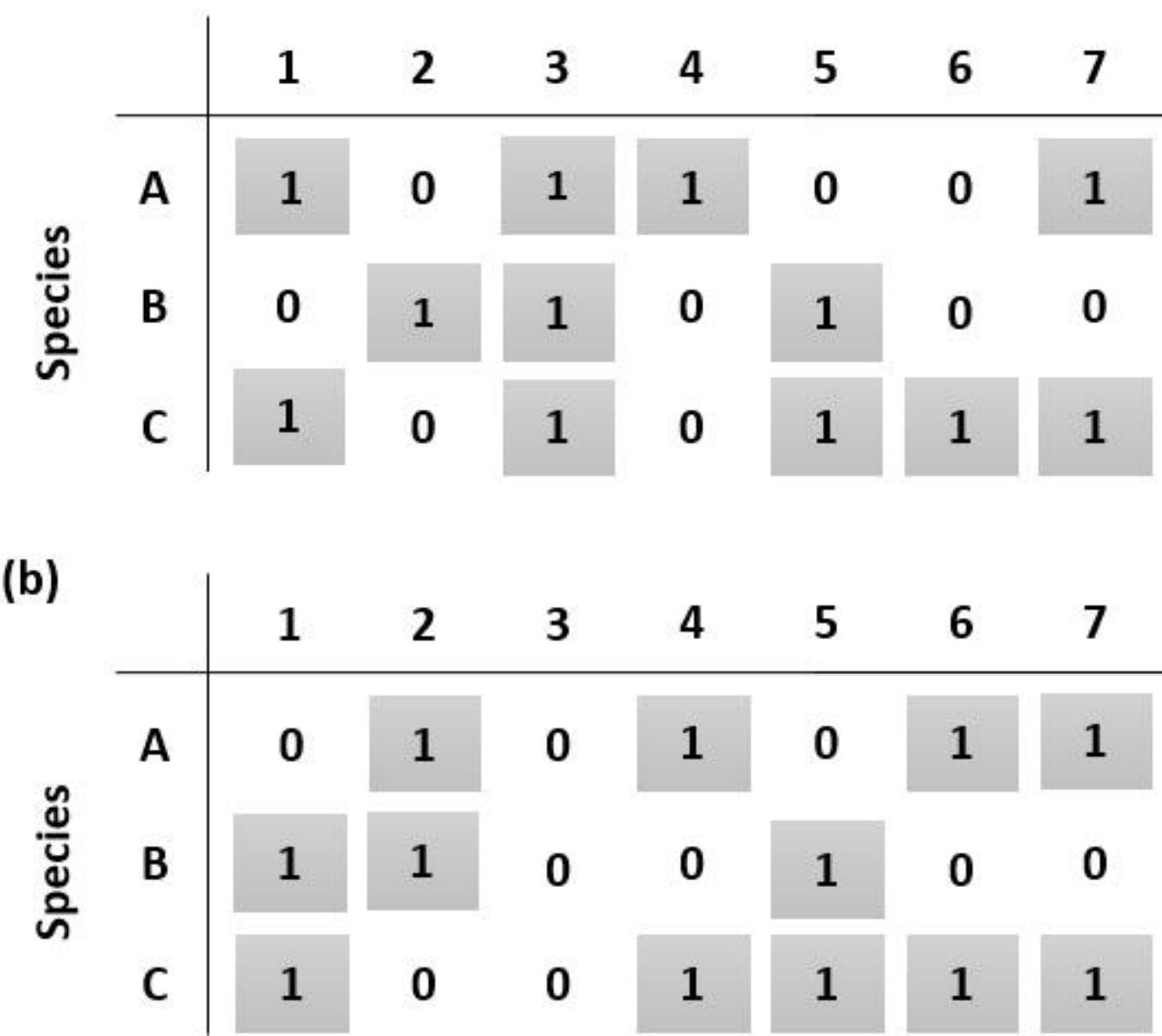

(b) 

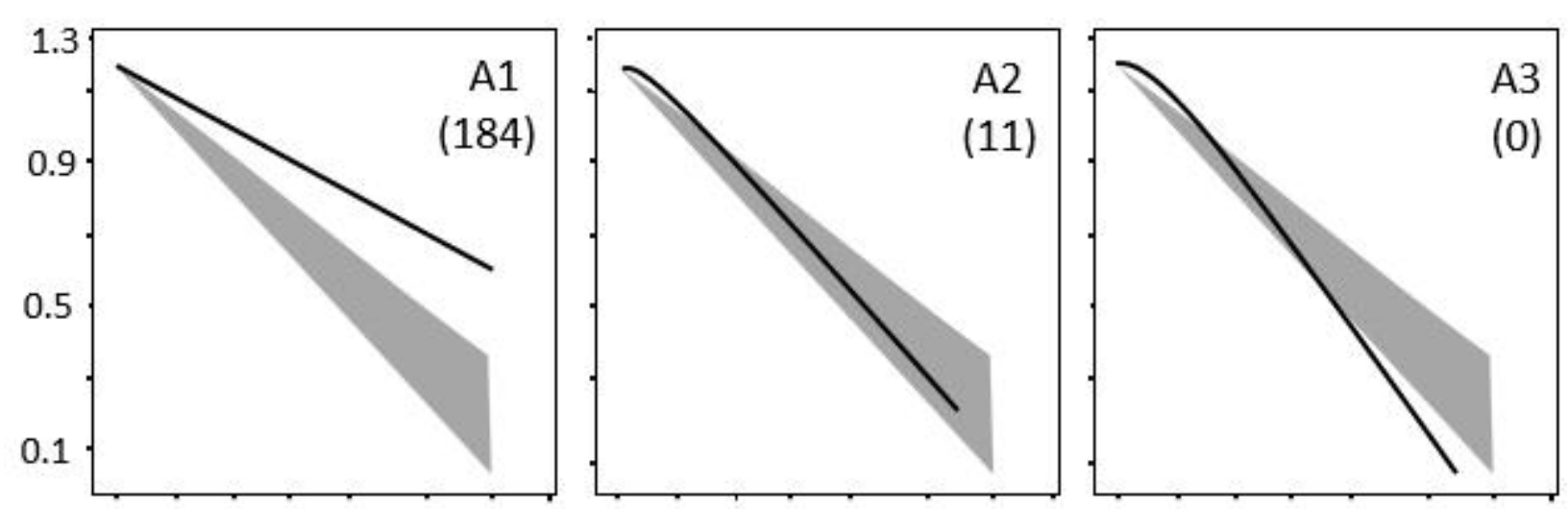

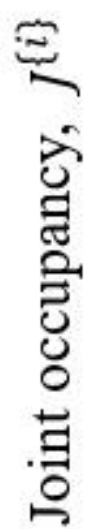
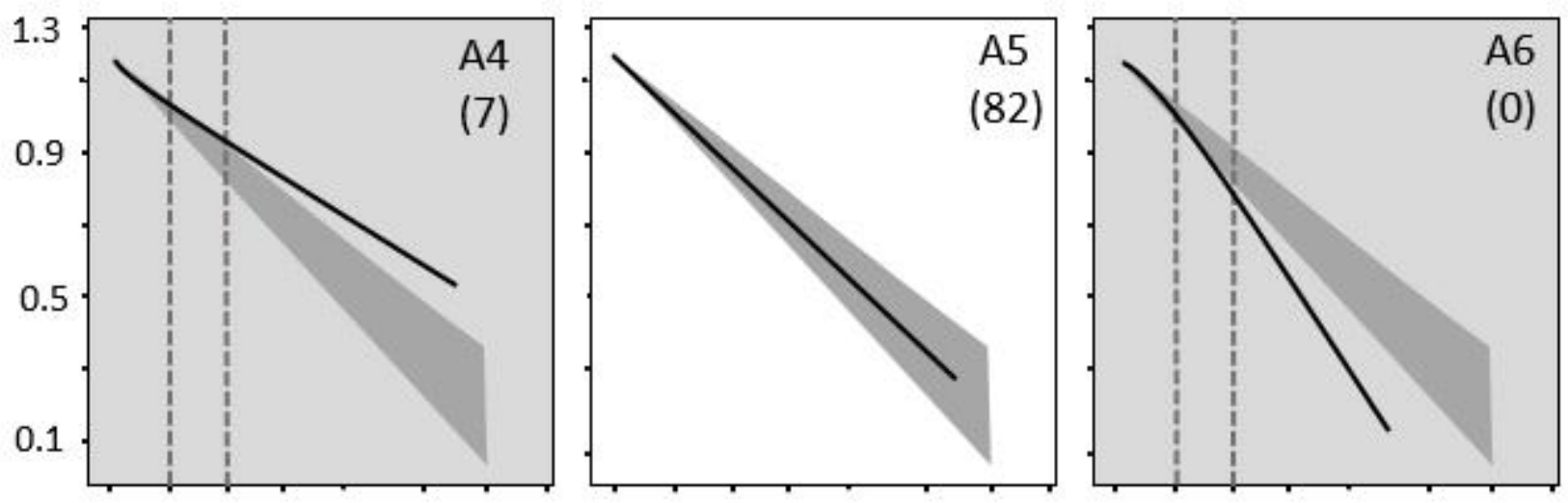

- Observed

Null model Type II error
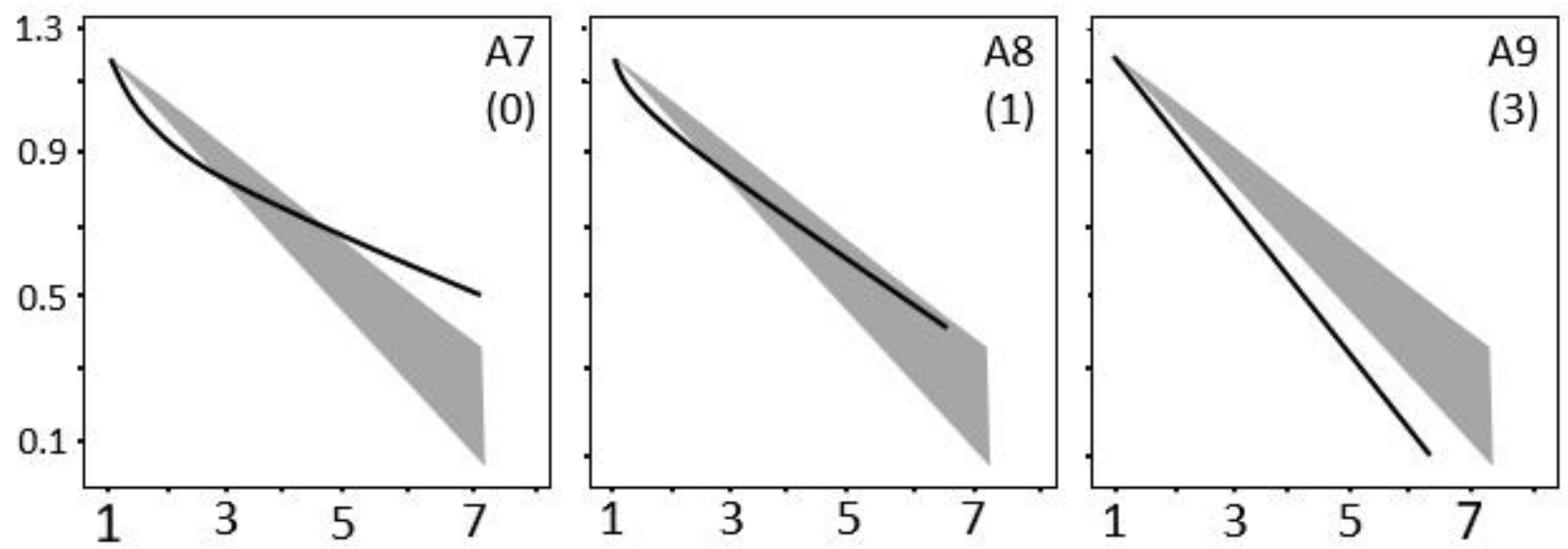

Number of species, $i$ 
